\title{
Práctica científica en América meridional. Experiencias sobre la velocidad del sonido en Santiago de Chile a fines del siglo XVIII ${ }^{1}$
}

\author{
Trinidad Larraín Donoso ${ }^{2}$
}

\begin{abstract}
RESUMEN
La renovación que desde hace algún tiempo han experimentado los estudios sobre historia de la ciencia, ha permitido que se comience a indagar en los procesos de expansión y difusión de la ciencia europea en otros espacios geográficos. Desde este enfoque, que ha puesto en evidencia el papel central que las prácticas científicas han tenido en las formas de producir conocimiento, este artículo aborda las experiencias sobre la velocidad del sonido realizadas en Santiago de Chile por los científicos ilustrados José Espinosa y Tello y Felipe Bauzá en enero de 1794. A través de un estudio de este experimento se analizan los métodos, procedimientos y herramientas utilizados por ambos oficiales españoles, con el propósito de reconstruir y conocer los modos de elaboración y validación del conocimiento científico en el espacio colonial iberoamericano, junto con demostrar que Chile no estuvo exento de actividad científica a finales del siglo XVIII.
\end{abstract}

Palabras clave: Historia de la ciencia, José Espinosa y Tello, Felipe Bauzá, práctica científica, siglo XVIII.

\begin{abstract}
The renovation that science history has been experiencing for some time now, has allowed the beginning of inquiry into the process of expansion and diffusion of European science in other geographical spaces. From this point of view, which has highlighted the central role that scientific practices have had on ways of producing knowledge, this article addresses the experiments carried out in Santiago, Chile regarding the speed of sound by the enlightened scientists José Espinoza y Tello and Felipe Bauzá in January of 1794. Through a study of this experiment we analyzed the methods, procedures and tools used by both Spanish officers, in order to reconstruct and understand the forms of elaboration and validation of scientific knowledge in colonial Spanish America, along with demonstrating that Chile was not exempt from scientific activity as of the end of the 18th century.
\end{abstract}

Key words: History of science, José Espinosa y Tello, Felipe Bauzá, scientific field work, 18 th century.

\footnotetext{
1 Preparado como parte de la investigación de la tesis doctoral "Un hidrógrafo de las dos América. La práctica científica del marino José Espinosa y Tello (1783-1809)", y en el contexto del grupo de trabajo del proyecto FONDECYT 1095221. En el artículo se utilizan las siguientes abreviaturas: AMN (Archivo del Museo Naval de Madrid), Ms (Manuscrito), f.
}

(Foja) y Doc. (Documento). Artículo recibido el 13 de marzo de 2012, aceptado el 22 de noviembre de 2012 y corregido el 31 de enero de 2013.

2 Programa de Doctorado, Instituto de Historia, Pontificia Universidad Católica de Chile (Chile). E-mail: tlarraindonoso@yahoo.es 
El estudio de las "Observaciones de la velocidad del sonido" que los oficiales de la Expedición Malaspina José Espinosa y Tello y Felipe Bauzá realizaron en el Llano del Maipo, en las cercanías de la ciudad de Santiago, entre los días 13 y 17 de enero de 1794, refleja el complejo proceso en la organización, elaboración, validación y circulación del conocimiento científico en el espacio colonial americano. Desde el enfoque que ha puesto en evidencia los procesos de expansión y difusión de la ciencia europea en otros espacios geográficos, vinculados al estudio de viajes de exploración científica y ciencia colonial (AA.VV, 1995; AA.VV, 1991; Peset y Lafuente, 1989; Revista de Indias, №180, 1987), nuestra propuesta indaga en los mecanismos de creación científica en un contexto donde la monarquía española basó sus políticas en el supuesto de que la adquisición y aplicación del conocimiento en América, incrementaría su poder político y económico en las posesiones ultramarinas (Nieto, 2006). En este sentido, la denominación de recursos, el control de rutas, la recolección de ejemplares, la construcción de colecciones, la experimentación física, las observaciones astronómicas, la representación del territorio, entre otras cosas, constituyeron actividades fundamentales del proceso expedicionario europeo que demostraron el afán ilustrado por trasladar y emplear nuevos conocimientos científicos al escenario natural americano.

Uno de los proyectos científicos de mayor envergadura que financió y promovió la corona española hacia sus territorios americanos fue la Expedición Malaspina. Comprendida entre los años 1789 y 1794, tuvo entre sus propósitos averiguar el estado político y comercial de las colonias; trazar derroteros para la navegación mercantil en todo el Pacífico; y levantar cartografía de los territorios visitados, con especial énfasis de las regiones fronterizas, sondeando sus posibilidades estratégicas y defensivas, sus habitantes y recursos naturales (Pimentel, 1998: 162-180, Sagredo y González, 2004).

En efecto, José Espinosa y Felipe Bauzá como miembros de esta empresa científica, a cargo de los ramos de Hidrografía y Cartografía respectivamente, exploraron, reconocieron, observaron y cartografiaron gran parte del imperio colonial español, redactando diarios y descripciones, levantando planos y mapas, apuntando cálculos astronómicos e hidrográficos, y llevando a cabo importantes reconocimientos geográficos como el relativo al paso de los Andes en el viaje de retorno (Higueras, 1987, 3 tomos).

Aquejados de escorbuto y asma, y por recomendación del propio comandante Alejandro Malaspina, los dos marinos españoles desembarcaron de las corbetas Atrevida y Descubierta en el puerto del Callao a fines de 1793, dirigiéndose primero a Chile, para luego atravesar la cordillera hasta Buenos Aires, y reencontrarse con los otros miembros de la expedición en Montevideo. El propósito de este viaje por tierra fue evitar el paso por el Cabo de Hornos y, de ese modo, restituir el estado de su salud (AMN, Ms. 314, f. 183). Sin embargo, este recorrido por el interior de América meridional significó un valioso aporte al conocimiento de "estos remotísimos países (...) tan desconocidos de los europeos" (Salazar, 1809: 67), enriqueciendo de manera muy singular los resultados obtenidos por la propia Expedición Malaspina. Lo anterior, puesto que se aportaron experiencias y trabajos de diversa índole, tales como la descripción del camino desde Valparaíso a Buenos Aires, variadas noticias sobre las costumbres, habitantes y naturaleza de aquellos territorios, recogida de datos acerca del sonido, la latitud, longitud y alturas barométricas, y haber levantado la "Carta esférica de la parte interior de América del Sur para manifestar el camino que conduce de Valparaíso a Buenos Aires, construido por las observaciones que se hicieron en estos parajes en 1794" (González, 2007: 25-27).

Las observaciones sobre la velocidad del sonido hechas por Espinosa y Bauzá en las cercanías de la ciudad de Santiago a fines del siglo XVIII, se explican y comprenden dentro de este contexto de reconocimiento y exploración de la fisonomía geográfica interior de América meridional. Ya antes que ellos, La Condamine apuntó la posibilidad de conocer la distancia de un punto a otro por medio de este tipo de experiencias (La Condamine, 1751: 36, 57 y 98). Como lo advierten Lafuente y Delgado, aquella idea era atractiva puesto que su aplicación "permitía efectuar "triangulaciones" que atravesaran montañas y lugares donde las condiciones climatológicas 
u orográficas fueran extremadas" (Lafuente y Delgado, 1984: 46). Motivado por aquellas ideas, Jorge Juan en compañía del astrónomo francés Louis Godin las practicaron entre agosto de 1737 y julio de 1738 en Ecuador, señalando que este tipo de experiencias además de la utilidad que obtenía de ellas la Física, podían destinarse a resolver algunos problemas de la geometría y la navegación (Juan y Ulloa, 1768: 132-143). Sin embargo, los resultados obtenidos por Juan, Godin y La Condamine demostraron la inviabilidad del método para la determinación de distancias, puesto que el procedimiento no garantizaba menos de 100 toesas en un grado de longitud (Lafuente y Delgado, 1984:47).

En conocimiento del experimento realizado por Juan, Espinosa y Bauzá decidieron resolver algunas cuestiones en torno al sonido que todavía no habían sido disipadas en Europa, acreditando sus propias experiencias a la teórica de Newton, como lo veremos en las páginas siguientes. Las palabras del propio Alejandro Malaspina son elocuentes en ese sentido, señalando que para él constituyeron unas "observaciones tan prolijas como importantes", y que "abrían un nuevo campo a esta clase de indagaciones físicas, hasta aquí no bien sujetadas a la Experiencia" (Novo y Colson, 1885: 330). Si bien es cierto no fueron los únicos que Ilevaron a cabo este tipo de observaciones durante el recorrido de la empresa científica en América -Antonio Pineda, naturalista de la expedición, también las realizó en el sitio El Arenal, en las inmediaciones del Chimborazo y en la provincia de Huarochiri, Perú (Galera, 1988: 192-195; Estrella et al., 1996: 85)- cabe destacar que la documentación generada por estos oficiales al respecto es única en cuanto al detalle, prolijidad e información que entregan sobre estas experiencias, lo que nos permite profundizar en los métodos, procedimientos y herramientas utilizados por Espinosa y Bauzá a la hora de efectuar este tipo de prácticas.

Como lo ha expresado Juan Pimentel, la noción de práctica científica vino a recordar algo tan esencial como que los científicos "no solo dicen que hacen cosas, sino que también las hacen" (Pimentel, 2010: 420). El poco interés que, en general, ha tenido la historiografía por el estudio de cómo los oficiales venidos en expediciones científicas han elaborado y construido el conocimiento en América, hace que este tipo de análisis comprenda un horizonte novedoso y poco trabajado que permite superar la visión que centra su mirada en los resultados más evidentes de los viajes, y que obliga a poner el acento en la producción del saber (Sagredo, 2008). Por lo tanto, comprender de qué manera el conocimiento fue generado y practicado en la América meridional a fines del siglo XVIII reviste de especial interés, por una parte, porque nos permite analizar los elementos y aparatos involucrados en este tipo de experiencias relacionadas con la exploración científica española. Además, dar cuenta cómo el conocimiento fue organizado y legitimado en América del Sur. Por último, intentar explicar en qué medida estas observaciones tuvieron importancia a nivel local, teniendo en cuenta la condición periférica en la que se encontraba Chile en esa época.

\section{Observaciones sobre la velocidad del sonido}

Fue a partir del siglo XVII que el interés por el estudio físico de la producción de los sonidos y su propagación despertó la atención de varios científicos europeos. Si bien desde la antigüedad se sabía que este se difundía a través del aire, existía la creencia que los distintos tonos tenían diferentes velocidades. Sin embargo, fueron los franceses Pierre Gassendi y Marino Marsenne quienes llevaron a cabo las primeras mediciones que determinaron que cualquier tipo de onda sonora, ya fuera grave o aguda, mantenía la misma constancia en su velocidad durante su recorrido. A partir de ese momento, se hicieron diversos ensayos y mediciones, estableciéndose fenómenos como la influencia del viento sobre la propagación del sonido, y que este se expandía igualmente en todo sentido, independientemente de su tono o intensidad (Shurmann, 1946: 522).

Sin embargo, no fue hasta la publicación en 1687 de los Principios Matemáticos de la Filosofía Natural de Newton que se consolidó el concepto matemático de la propagación del sonido y su cálculo. El interés por el movimiento de los cuerpos y la mecánica de 
las vibraciones en un medio elástico llevó al célebre científico inglés a relacionarse con la acústica, y de ese modo, plantear en el segundo libro de sus Principios la primera fórmula para calcular la velocidad del sonido en el aire, que según este en condiciones normales de temperatura correspondía a 280 $\mathrm{m} / \mathrm{s}$, cuando hoy sabemos que al aplicar la ley adiabática de los gases su valor correcto es de $342 \mathrm{~m} / \mathrm{s}$ (Newton, 1987: 438; Garrido, 1996: 237-238). No obstante, estos resultados no coincidieron con muchas de las experiencias hechas por diversos físicos y matemáticos de la época y posteriores a Newton, que entrado el siglo XVIII intentaron una y otra vez comprobar y resolver el problema de la velocidad del sonido obteniendo valores menores a la fórmula newtoniana respecto a los cálculos que arrojaban sus procedimientos experimentales (Shurmann, 1946: 522-524; Beyer, 1999: 4-6).

Las experiencias hechas por los oficiales José Espinosa y Felipe Bauzá en las cercanías de la ciudad de Santiago a fines del siglo XVIII, posiblemente tuvieron su origen en la inquietud científica debida a los estudios sobre la propagación de ondas para determinar la velocidad del sonido en el aire de Newton, puesto que sus conjeturas se mantuvieron vigentes durante más de un siglo. Asimismo, científicos como William Derham planteaban dudas respecto a si la velocidad del sonido era la misma en cualquier altura sobre la superficie de la Tierra y si este se movía en iguales velocidades en las distintas regiones, es decir, en los climas septentrionales y meridionales. Hasta ese momento, las mediciones en zonas más tórridas que se habían llevado a cabo eran las de Jorge Juan, Godin y La Condamine en Quito (Juan, 1768: 133-135, 140). Pensamos que el paso por una de las colonias más australes del imperio español, dio la oportunidad a Espinosa y Bauzá de contestar a estas preguntas, teniendo en cuenta la posición geográfica en la cual se hallaba la capital del Reino de Chile ${ }^{3}$.

\footnotetext{
3 Según las propias observaciones hechas por Espinosa y Bauzá en 1794, determinaron que la latitud de Santiago era de $33^{\circ} 26^{\prime} 1 / 3$ Sur y su longitud de $70^{\circ}$ $43^{\prime}$ O. de Greenwich. AMN., Ms.579, f. 64.
}

Unido a la inclinación natural que estos marinos sentían por el conocimiento, producto además de un contexto ilustrado, en las primeras líneas de las "Observaciones sobre la velocidad del sonido" se desprende el carácter heroico que ambos quisieron otorgarle a sus experiencias. Aunque estos reconocen que en diversas épocas y lugares se habían efectuado estas observaciones, con vistas a "los progresos de la física general", advierten que "animados por el mismo espíritu" realizaron "las siguientes en el Ilano de Maypó del reyno de Chile, donde no se habían practicado hasta ahora" (Espinosa y Bauzá, 1809: 170). De aquella aseveración hecha por Espinosa y Bauzá se desprende la idea de que ambos oficiales intentaron acentuar sus logros científicos bajo el carácter de haIlazgo. El proclamar que nadie había hecho antes este tipo de experiencias en la colonia más austral del imperio, no solo demuestra la intención heroica de ser reconocidos por esa proeza, sino también una actitud de derecho de posesión y dominio sobre el fenómeno descubierto (Nieto, 2009: 62 y ss.). Espinosa y Bauzá al "hallar lo que estaba ignorado" (Diccionario de la Lengua Española, 1992: 705), tuvieron que transformar ese algo desconocido en un saber familiar y cierto, lo que implicó necesariamente un proceso de construcción de ese conocimiento a través de un lenguaje, códigos y prácticas propios de la ciencia ilustrada.

Si bien es cierto que en el pensamiento ilustrado español el saber tenía como fin facilitar a la vida humana nuevos descubrimientos y mayor poderío, lo que demostraba el carácter fabril y utilitario de la ciencia, la idea del conocimiento basada en la observación y experimentación como medio de comprensión de la naturaleza y sus fenómenos llevó a que se sucedieran teorías y experimentos que, en definitiva, legitimaron la actividad científica (Gay, 1983: 16-17).

En ese escenario, y como bien lo señalan Steve Shapin y Simon Schaffer, el experimento surgió como un medio sistemático para generar conocimiento sobre el universo, donde las "prácticas científicas se institucionalizaron y los hechos producidos experimentalmente devinieron en fundamentos de lo que contaba como conocimiento científico apropiado" (Shapin y Shaffer, 2005: 30). De 
esa forma, las prácticas experimentales cobraron un nuevo valor en la producción de conocimiento confiable, aunque nunca estuvieron exentas de críticas por parte de quienes negaban y ponían en duda su estatuto epistemológico ${ }^{4}$.

El examen del experimento Ilevado a cabo por José Espinosa y Felipe Bauzá en 1794, entonces, no solo explica la manera en que fueron producidos los hechos experimentales, sino también vislumbra cómo el conocimiento fue organizado y legitimado en la América colonial.

\section{Práctica científica: experimentos, instrumentos y cálculos}

Como lo demuestra la lectura de las "Observaciones sobre la velocidad del sonido", el texto se divide fundamentalmente en dos partes. La primera de estas titulada De la velocidad del Sonido, constituye una síntesis general que aborda el problema del sonido a partir de las observaciones practicadas por otros científicos. A través de los resultados obtenidos por estos, ambos marinos exponen el origen de este, sus principales características y variaciones. El segundo apartado, Experiencias de la velocidad del sonido, describe el experimento realizado por los dos oficiales en Chile, explicando el principio sobre el cual fundamentaron la observación, los instrumentos utilizados, las operaciones Ilevadas a cabo y los resultados obtenidos (Espinosa y Bauzá, 1809: 169-177).

Para determinar la velocidad del sonido ambos oficiales especifican que se basaron en el siguiente principio: "Quando se ve la explosión de un arma de fuego á alguna distancia, percibe la vista la luz de la pólvora inflamada mucho tiempo antes que se

\footnotetext{
4 Ejemplo de lo señalado fue la controversia ocurrida en Inglaterra en la década del 1660 y principios de 1670 entre Robert Boyle y Thomas Hobbes, donde el primero aparece como el mayor practicante de la experimentación sistemática y uno de los más importantes propagandistas del valor de las prácticas experimentales en la filosofía natural y Hobbes como el oponente local más ferviente, tema que traspasa el libro de Shapin y Shaffer.
}

oiga el sonido; y como la luz se propaga con tanta rapidez, que no tarda dos segundos en venir de la Luna á la tierra, puede decirse que la vista la percibe en el mismo momento que sale del cuerpo sonoro, en vez de que el estrépito producido al propio tiempo por este, emplea en llegar al órgano del oído un tiempo sensible y determinado". A lo que agregan: "Luego midiendo con precisión este tiempo y la distancia del observador al sitio donde se produce el sonido, se sabrá la velocidad actual de este" (Espinosa y Bauzá, 1809: 171).

Podemos advertir que el modelo experimental utilizado para la medición de la velocidad del sonido fue simple, pues consistió en la estimación del tiempo transcurrido entre la percepción visual de la explosión producida por la detonación de un arma y la percepción auditiva del disparo, junto al cálculo del espacio entre el observador y el paraje del suceso ${ }^{5}$. Pero además se vislumbra que aquellas mediciones debían ser hechas "con precisión", es decir, siguiendo a Nuria Valverde, bajo un lenguaje y valores comunes, asociados al uso y posesión de instrumentos científicos, que además de exactitud matemática, posibilitaran la homogenización, fiabilidad y consenso sobre los modos de comunicación e intercambio del conocimiento (Valverde, 2007: 123 y 124).

Por lo anterior, no resulta extraño que Espinosa y Bauzá, al narrar el experimento llevado a cabo, detallaran en este la utilización de los medios mecánicos que les permitieron establecer de manera certera y exacta el tiempo de los intervalos señalados: "Para la medida del tiempo nos valimos de dos buenos reloxes de segundos, ajustados al movimiento medio por observaciones del Sol, y comparados freqüentemente á dos péndulos de medios segundos que construimos, y usamos ya uno ya otro de estos instrumentos

\footnotetext{
5 Este procedimiento experimental fue el más común para averiguar la velocidad del sonido. Lo usó Pierre Gassendi en sus experiencias de 1624, lo mismo Jorge Juan en América. A su vez en el Tratado de navegación por Don Josef de Mendoza y Ríos, Teniente de Navío de la Real Armada, Tomo Segundo, 1787:153-154, el marino reconoce que este es el método más idóneo para llevar a cabo este tipo de experiencias. Shurmann, 520; Juan, 135-139.
} 
en las experiencias para que fuesen más independientes los resultados" (Espinosa y Bauzá, 1809:171).

Además de la utilización de ciertos instrumentos científicos para conseguir la precisión deseada, las observaciones que llevaron a cabo Espinosa y Bauza, como ellos mismos lo indican, las hicieron en "varias direcciones y á diversas distancias, desde dos hasta ocho millas", con el objeto de poder compararlas entre sí y así obtener resultados más autónomos (Espinosa y Bauzá, 1809: 171). De hecho, los oficiales se trasladaron a distintos puntos aledaños a la ciudad de Santiago a practicar sus experiencias.

Figura $\mathrm{N}^{\circ} 1$

Triangulación que representa la base medida $\mathrm{ab}$, y las distancias $\mathrm{c}, \mathrm{d}, \mathrm{e}, \mathrm{f}$ del Ilano al extremo $b$.

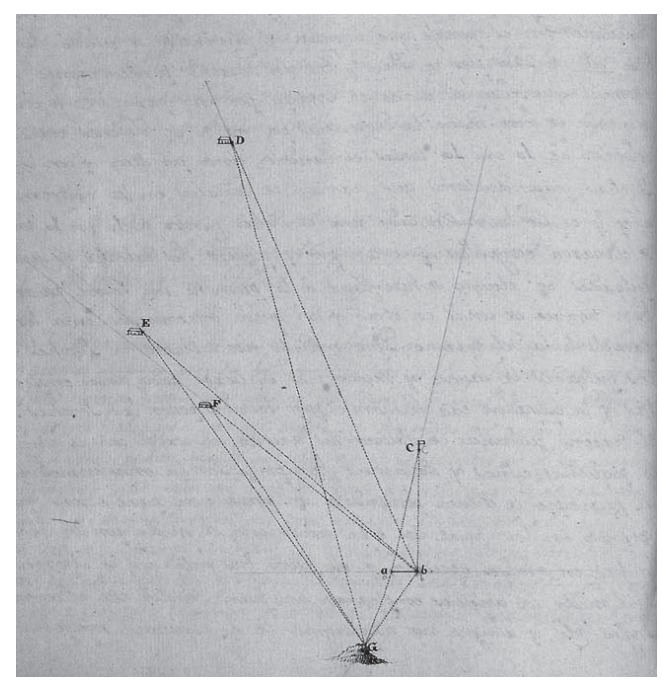

Fuente: Archivo Museo Naval de Madrid.

La Figura $N^{\circ} 1$, muestra la triangulación geodésica propuesta para la determinación de la distancia y posición de los distintos lugares designados por los marinos para realizar las observaciones correspondientes. Así podemos visualizar que fue el punto B donde se decidió iniciar la medición respecto a los otros puntos, y donde se puso el cañón para hacer los disparos correspondientes. Este equivale al sitio de la ex chacra de El Conventillo, lugar situado en la zona sur de Santiago.
Por su parte, el vértice $\mathrm{C}$ se refiere a un palo ubicado hacia el SO del lugar del Conventillo. En cuanto a los vértices D, E y $F$ representan respectivamente las chacras del Peral, la de Cañas y la de Macul, todas extensiones ubicadas en la zona periférica sur oriente de Santiago, lo que actualmente conocemos como las comunas de La Florida y Puente Alto. Por último, el punto G, y como la misma imagen lo muestra, corresponde al cerro Santa Lucía (AMN, Ms 579, f.71v).

También la imagen de los triángulos representa la base medida AB. Como parte del procedimiento empleado en la triangulación geodésica, primeramente se medía una determinada longitud de terreno, que se denominaba base fundamental. Esta se calculaba de manera sencilla - "la operación era facilísima" - pero muy cuidadosa "por medio de piques verticales colocados de 10 en 10 toesas, enfilándolos unos por otros y con dos perchas perpendiculares, que servían de señales en los extremos A y B de la base" (Espinosa y Bauzá, 1809:172). De ese modo, Espinosa y Bauzá en los puntos de 10 en 10 toesas, es decir, 19,46 metros aproximadamente, clavaron estacas de un pie dejando sus extremos a cuatro pulgadas de altura del suelo con el propósito de pasar desde la primera hasta la última de estas un cordel "bien tirante", que fijaba una línea paralela a la del terreno. Una vez hecha esta traza, los oficiales pasaron a establecer su extensión ${ }^{6}$.

La finalidad de establecer la distancia de una base fundamental de antemano, en este caso una de 2.900 pies de París, que equivale hoy en día a unos 941 metros, obedecía a que esta se constituía en un lado del primero de una serie de triángulos encadenados,

6 Según Jorge Juan la toesa equivalía a unos 6 pies de París. Lo anterior también es afirmado por Dionisio Alcalá Galiano, oficial de la Expedición Malaspina. Según el sistema de equivalencia que propone Nuria Valverde, "una vara tenía una longitud de 0.835905 metros. El pie, dado que era la tercera parte de una vara, equivalía entonces a 27,8 centímetros. La vara de Madrid era más larga $(0,843 \mathrm{~m})$, por lo que su pie era de $28,1 \mathrm{~cm}$. Según Jorge Juan una vara era $371 / 144$ pies de París, por lo tanto este equivalía a unos $32,44 \mathrm{~cm}$. Con todo lo señalado, una toesa correspondía aproximadamente a 194, $64 \mathrm{~cm}$ o 1,9464 m. (Valverde, 2007:195, Juan, 1768: VII, Alcalá Galiano, 1809:183). 
como la misma imagen lo muestra. A partir del conocimiento de aquella longitud, sumado al conocimiento de los tres ángulos interiores del triángulo, podía calcularse la extensión de otro de los lados de dicho triángulo, que a su vez pasaba a ser la base del segundo triángulo de la serie, y así sucesivamente (SeIlés, 2000: 256).

Para obtener los ángulos, Espinosa y Bauzá señalan en sus "Observaciones..." que emplearon "un buen teodolite de Ramsden". Esta afirmación de los oficiales sobre el uso de ese instrumental resulta dudosa por algunas razones que pasaremos a comentar, aunque explica, en gran medida, la relevancia y el grado de legitimidad que estos aparatos otorgaban a la actividad científica a fines del siglo XVIII.

Al revisar las distintas versiones y copias que existen de las "Observaciones..." en el Museo Naval de Madrid, podemos corroborar lo enunciado, ya que en estas se leen que el teodolito utilizado por Espinosa y Bauzá en las mediciones de ángulos fue el inscrito por el inglés Humphrey Cole, y no el construido por Ramsden (AMN, Ms. 579, 59v, 69, 71, 72, 152v).

¿Qué lleva a estos oficiales científicos a estipular en la publicación de las "Observaciones..." que usan un tipo de teodolito en la medición de los ángulos y no el que verdaderamente utilizaron? ¿Tiene alguna importancia nombrar un instrumento y no otro? ¿Cuál es la función retórica que cumplen estos instrumentos de medición dentro de las prácticas científicas?

Como lo ha expuesto Nuria Valverde, la búsqueda de precisión a través del uso de los instrumentos científicos, más que un problema matemático y técnico, que sí era importante, se justificaba, en gran medida, al papel social y público que deseaban ejercer los científicos (Valverde, 2007: 123). Espinosa y Bauzá al incorporar en su relato la especificación de ciertos artefactos empleados en sus experiencias, no hicieron más que consolidar un discurso científico que determinaba aquellos aspectos de la realidad que les permitieron formar parte de una comunidad científica ${ }^{7}$. Como los instrumentos científicos creaban un vínculo incuestionable entre el objeto que medían y la cifra obtenida, que era dado por la reputación del artífice del instrumento, los oficiales ilustrados al especificar el uso del teodolito de Ramsden no hicieron más que destacar con ese antecedente la fiabilidad de los resultados obtenidos en sus experiencias, ya que a fines del siglo XVIII cualquier observación o experimento que no estuviese medido por una tecnología gozaba de escasa legitimidad (Lafuente y Valverde, 2003: 97-98, 102).

La construcción y producción del conocimiento necesitaba estar ligada a ciertas prácticas y usos, que eran las que consolidaban una retórica común. Eran esas prácticas y esos usos los que finalmente legitimaban la actividad científica de los marinos ilustrados, además de entregarles reconocimiento, voz y visibilidad como lo demuestra el caso de estas "Observaciones...". En ese sentido, el señalar que emplearon un equipamiento más moderno y exacto del que verdaderamente usaron, obedece no solo a la transferencia de prestigio que estos le entregaban a los experimentos, sino también a una forma determinada de crear información, ligada a ciertas tradiciones, consensos y acuerdos tácitos dentro de la ciencia ilustrada (Valverde, 2007: 194). Este lenguaje común, cimentado en la precisión de los registros, en la utilización de artefactos científicos para la realización de experimentos y en la objetividad del conocimiento adquirido a través de estos, era el que configuraba unas redes amplias y estables que hacían que el trabajo científico perdiera

\footnotetext{
7 Respecto al concepto de comunidad científica Nuria Valverde señala que "entre finales del siglo XVII y principios del siglo XVIII los científicos comienzan a consolidarse como comunidad, y a adquirir presencia social. Se los identifica como productores de un saber universal garantizado por el juicio crítico de los miembros de esta comunidad desinteresada a la que se denominó la República de las Letras. Gracias a la vigencia de determinados códigos de cortesía o civilidad, que regulaban la transacción de la información, durante un largo período se pudo sostener el entramado de relaciones que caracterizaba a este modo de gobierno del ingenio. Progresivamente, sobre todo a partir de 1720, dichos códigos evolucionaron para garantizar que la comunicación respetara determinadas normas de control" (Valverde, 2007: 1)
} 
su carácter local y se hiciera Universal (Nieto, 2009: 184). Por lo tanto, los instrumentos científicos constituyeron un elemento imprescindible dentro de la retórica e identidad del viajero científico ilustrado, puesto que develaron el desarrollo de sofisticados artefactos y de tecnología de precisión, con los cuales se podía producir avances teóricos importantes en los distintos ámbitos del saber (Lafuente y Valverde, 2003: 94 y ss.).

Prueba de lo señalado fue la preocupación que tuvo Alejandro Malaspina de conseguir para su viaje instrumentos científicos que le aseguraran en la práctica los más avanzados métodos de cálculos astronómicos en la determinaciones de latitud y longitud, en las observaciones de inclinación de aguja, las observaciones de la variación magnética y barométricas, entre otras preocupaciones. Muchos de estos aparatos fueron encargados al extranjero a los mejores artífices de la época, entre ellos Ramsden, Wright, Stanclif y Trougthon, $y$ otros fabricados expresamente para la expedición (Glick, 1989: 53 y ss.). Del mismo modo, el documento "Instrucciones para los trabajos científicos" redactado por el comandante italiano en agosto de 1790, daba cuenta del propósito de este de unificar bajo un mismo método la anotación de cálculos y observaciones que se hicieran a lo largo de la empresa científica, para así establecer un conocimiento confiable y útil (Higueras, 1987, Tomo II: 25-27).

Volviendo al experimento, Espinosa y Bauzá al poseer ciertos datos, en este caso, la medida de la base fundamental $A B$ y los ángulos interiores de cada triángulo, pudieron someter esa información a un proceso de cálculos trigonométricos que les permitió tornarlos válidos dentro de la comunidad científica, además de resolver el problema de la distancia que recorre el sonido en un segundo, fundamental para concluir la velocidad de este (Sellés, 1992: 13). Por lo tanto, el conocimiento de trigonometría plana les permitió a Espinosa y Bauzá calcular distancias que no podían medir directamente, sino que solo a través de estos procedimientos matemáticos ${ }^{8}$.

\footnotetext{
8 La formación de Espinosa y Bauzá en la Compañía de Guardias Marinas de Cádiz y su paso por el
}

Ejemplo de lo expuesto son los documentos (Figura $N^{\circ} 2$ ) que muestran los cálculos trigonométricos hechos para obtener las medidas de los distintos lados de los triángulos, que equivalían a las respectivas distancias desde donde los marinos hicieron sus experiencias. En estos se aprecia, en primer lugar, que en el lado derecho de ambos manuscritos se hallan anotados los ángulos y minutos observados por medio del teodolito. En segundo lugar se pueden observar los cálculos de cada uno de los triángulos, ya que con los ángulos observados de todos estos y la base $A B$ de 2900 pies de parís -que según el cálculo de su logaritmo era igual a 3,4623980-, se dedujo el valor de todos los lados de la serie de triángulos, para con ellos determinar la distancia respecto a lo distintos puntos de observación de las experiencias.

Los cinco triángulos utilizados para conocer las distancias -que en realidad son diez, puesto que la pretensión de precisión de Espinosa y Bauzá en sus cálculos los llevó a comprobar las medidas obtenidas en un mayor número de triángulos encadenados-, permitieron concluir a los oficiales científicos las distintas longitudes de estas "con todo el rigor de la geometría", que según sus cálculos fueron "(...) CB de 13.841 pies; EB de 43.365; GB de 9.557; FB de 29.558, y DB de 50.316" (Espinosa y Bauzá, 1809:172). Por lo tanto, el uso de logaritmos en sus cálculos trigonométricos les proporcionó aminorar gran parte del trabajo relacionado con ellos, puesto que los ayudó a simplificar las operaciones de multiplicación, división, elevación a potencias y extracción de raíces que conllevaban estos tipos de ejercicios (Granville, 1950: 160-165).

Concluidas estas operaciones trigonométricas, Espinosa y Bauzá se trasladaron al sitio del Conventillo y como lo relatan en sus

Curso de Estudios Mayores, significó que ambos oficiales se adiestraran en el uso de los métodos para la determinación de la longitud en el mar, la utilización de instrumental científico adecuado y la práctica del método de las distancias lunares. Lo anterior implicó que tuvieran profundos conocimientos de astronomía y manejo de la trigonometría esférica y plana, y de los tratados náuticos de la época (Lafuente y Sellés: 1989:485-504; Sellés, 2000; Sagredo, 2008). 
Figura $\mathrm{N}^{\circ} 2$

Mediciones trigonométricas para calcular la velocidad del sonido en Santiago de Chile en 1794

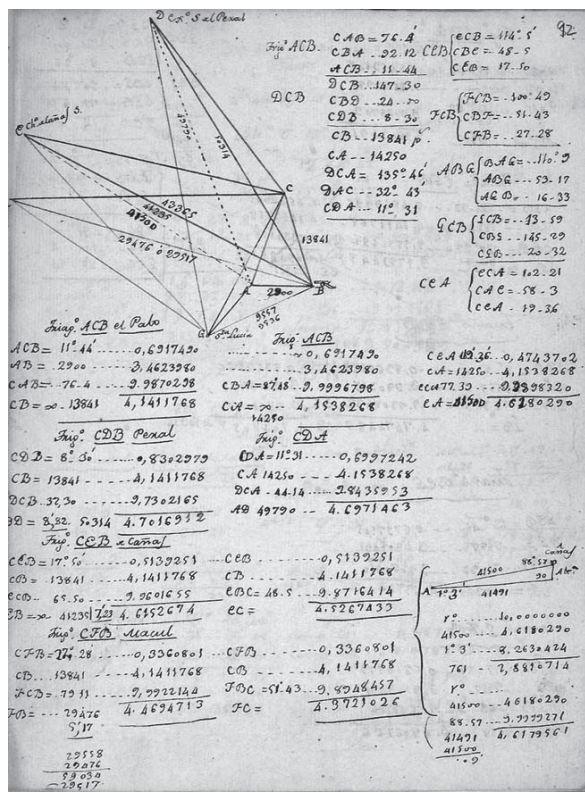

Fuente: Museo Naval de Madrid.

"Observaciones..." colocaron "un cañón de á ocho, y se dio principio á las experiencias" (Espinosa y Bauzá, 1809: 172). Vemos de esa forma, que la metodología seguida por los marinos comprendió dos etapas: la primera propiamente trigonométrica, es decir, el cálculo mediante triangulaciones sucesivas de la longitud de las diversas distancias establecidas, para así evitar errores derivados de una sola medición; y una segunda fase que consistió en llevar a cabo el experimento propiamente tal, mediante la observación y constatación del tiempo que se demoraba el sonido en recorrer el espacio determinado.

El protocolo utilizado por Espinosa y Bauzá en la recolección de los datos obtenidos obedeció al uso de tablas que expusieran con claridad las medidas de tiempo tomadas entre el tiro del cañón, la luz de este y el momento de su audición. Los oficiales registraron con prolijidad no solo el intervalo de tiempo en minutos y segundos, sino también el día y la hora en que fueron hechas las observaciones, además de las condiciones atmosféricas -el estado del viento, la presión del lugar a través de la medición del barómetro y la temperatura del ambiente por medio del termómetro-,

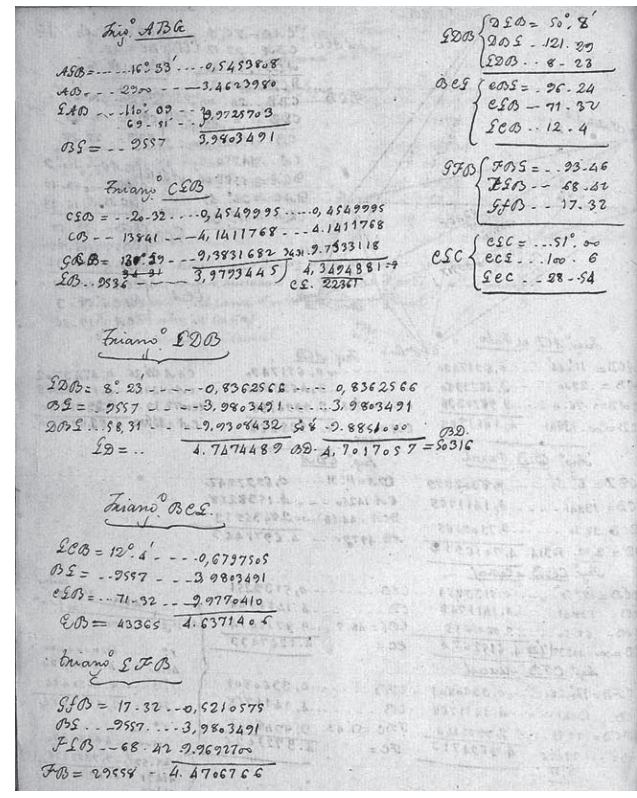

toda información esencial para el cálculo de la velocidad del sonido, como se puede apreciar en los documentos en que constan sus experiencias (Figura $N^{\circ} 3$ ) .

El experimento fue hecho "por medio de cuatro series de experiencias exactas en diversas direcciones" (AMN, Ms. 579, f. 63). Al anotar esta información, los marinos ilustrados no buscaron demostrar alguna hipótesis, sino comunicar el saber de una forma que les permitiera participar de ciertos consensos y códigos dentro de la comunidad científica. Al recoger este tipo de datos por medio de la observación directa, sumado al uso de instrumental técnico, entraron a un protocolo de adquisición de información consensuado por la ciencia ilustrada, que a su vez era normalizado y legitimado, puesto que la información ahí contenida era equiparable con otros experimentos del mismo ámbito. Existe, entonces, una intención de unificar el conocimiento que obedecía a una voluntad de homologación relacionada con cierta forma de hacer las observaciones, que demostraba el compromiso de la ciencia de ser replicable para poder ser legítima (Lafuente y Valverde, 2003: 101-102). 
Figura $\mathrm{N}^{\circ} 3$

Experiencias sobre la velocidad del sonido en Santiago de Chile 1794.

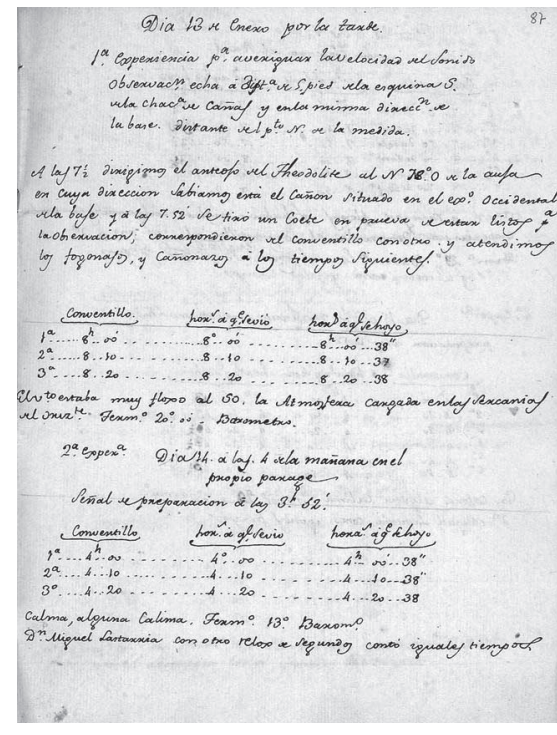

Fuente: Archivo Museo Naval de Madrid.

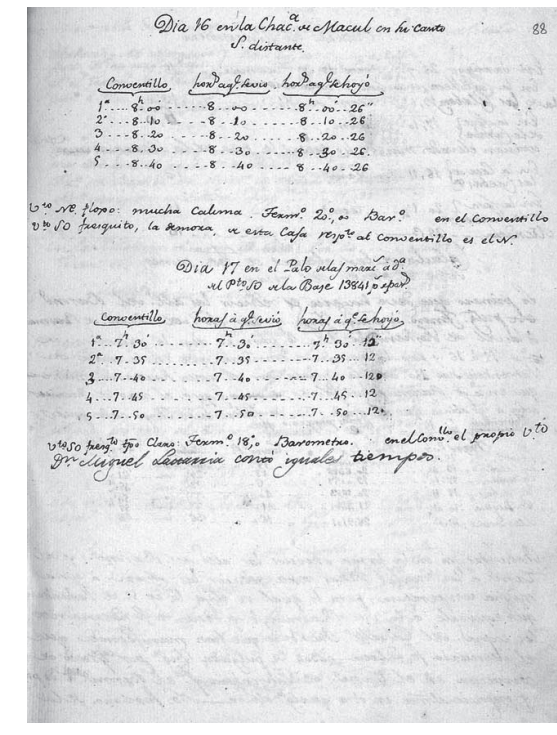

por una parte, inconvenientes en la anotación de los cálculos, y por otro, entregar una explicación clara y exacta de las mismas. Al poseer aquel conocimiento una misma óptica, se hacía compatible con diferentes tipos de información, lo que justificaba una forma particular de codificar, colectar, calcular y difundir los datos sobre los fenómenos de la naturaleza americana a fines del siglo XVIII (Galera, 2003: 8).

Por lo mismo, José Espinosa y Felipe Bauzá no dudan en especificar que "todas las experiencias que anteceden son de entera confianza", puesto que al ser partícipes de los consensos y protocolos promovidos por el pensamiento ilustrado la información que entregaban era verdadera. A pesar de lo señalado, ambos oficiales reconocen abiertamente un cierto grado de inexactitud en las mismas: "examinándolas con cuidado se ve que en la determinación del tiempo cabe quando mas medio segundo de error, que repartido en el número de las hechas en cada lugar es un error despreciable", agregando que "el viento solo pudo tener algún influxo en la quarta experiencia, retardando el sonido media toesa por segundo: en las experiencias restantes ó no hubo viento, ó tablas y lenguajes especializados, evitando, 
fue exactamente transversal y siempre floxo" (Espinosa y Bauzá, 1809: 175).

Lo anterior demuestra que los pequeños errores que pudieran darse dentro de las prácticas experimentales no eran fundamentales a la hora de evaluar si ese conocimiento era o no válido. Si bien estas inexactitudes podían afectar el resultado final de los datos obtenidos y de esa manera tornarlos inválidos, interesaba más que el conocimiento estuviera sujeto a ciertos modos de producción legitimados por la comunidad científica ilustrada, las academias de ciencias, únicas instituciones, social, profesional y administrativamente capaces de validar el conocimiento científico (Hernández, 2008: 145).

Para obtener la velocidad del sonido los marinos realizaron una operación matemática simple que consistió en dividir "el número de toesas que distan cada uno de los puntos C, D, E, F del punto B por el número de segundos que tardó el sonido desde el cañón al observador", determinando que la velocidad media del sonido en Chile fue de 191 toesas por segundo equivalente a $372 \mathrm{~m} / \mathrm{s}^{9}$.

Si bien Espinosa y Bauzá habían obtenido las distancias en pies de parís, al momento de determinar la velocidad del sonido estas fueron reducidas a toesas, puesto que esta era una unidad de medida francesa estándar que se mantuvo como referencia hasta la Revolución Francesa, momento en que se consolidó el sistema métrico universal (Hernández, 2008: 150). Aquella situación, desde la perspectiva ilustrada, nos habla de la importancia del uso de unidades de medidas estandarizadas a la hora de comunicar el conocimiento, puesto que sin estas era difícil la realización de comparaciones dentro del mundo científico europeo. Como medida de longitud, la toesa permitió a los científicos ilustrados el intercambio y contraste de sus resultados con los de otros científicos que habían hecho este mismo tipo de experiencias. Ejemplo de lo manifestado, es que Espinosa y Bauzá compararon sus experiencias con las observaciones que hizo Jorge Juan en Quito y Cassini en

\footnotetext{
9 La suma de todas las velocidades halladas da un total de 762,8 , resultado que divido en 4 es igual a 190,7, por lo tanto en su aproximación 191.
}

París, los cuales obtuvieron sus resultados en toesas (Espinosa y Bauzá, 1809: 175).

Por lo tanto, el conocimiento ilustrado español demandó esta transferencia con el objeto de cumplir con las mayores exigencias de precisión, donde este patrón de medida y sus equivalencias fueron empleados no solo como un referente unificador dentro de la comunidad científica, sino también como parte fundamental del discurso de los viajeros ilustrados, que como José Espinosa y Felipe Bauzá, calibraron el fenómeno de la velocidad del sonido. La concreción de un lenguaje científico especializado, la habilidad de utilizar el instrumental necesario para medir los fenómenos naturales con precisión y la institucionalización de las prácticas científicas fueron elementos esenciales dentro del universo científico del siglo XVIII, lo que implicó que la actividad científica estuviera cada vez más consolidada respecto a lo que se consideraba como conocimiento.

\section{Experimento y texto: formas de conocer y de dar a conocer}

Las experiencias sobre la velocidad del sonido hechas por Espinosa y Bauzá nos permiten indagar, por otra parte, en temas como la generación de autoridad científica por medio del análisis de la relación entre el experimento en sí y el texto que lo narra, pues el conocimiento para que adquiera autoridad necesariamente tiene que ser proclamado y reconocido por la comunidad científica (Nieto, 2009:68).

En esa línea, los diversos borradores que de este escrito se hallan en el Archivo del Museo Naval de Madrid (Figura N4), los cuales están Ilenos de notas y apuntes, muestran en primer lugar el interés de José Espinosa y Felipe Bauzá, de redactar un texto definitivo con el fin de ser publicado y dado a conocer a la comunidad científica tanto española como europea. De hecho, el escrito vio la luz por primera vez como apéndice de las Memorias sobre las observaciones astronómicas, hechas por los navegantes españoles en distintos lugares del globo..., edición a cargo del propio José Espinosa y publicado en Madrid por la Dirección de Hidrografía en 1809. 
Figura $\mathrm{N}^{\circ} 4$

Borrador de las "Observaciones de la velocidad del sonido, de latitud, longitud y variación hechas en Santiago de Chile por los oficiales Bauzá y Espinosa"

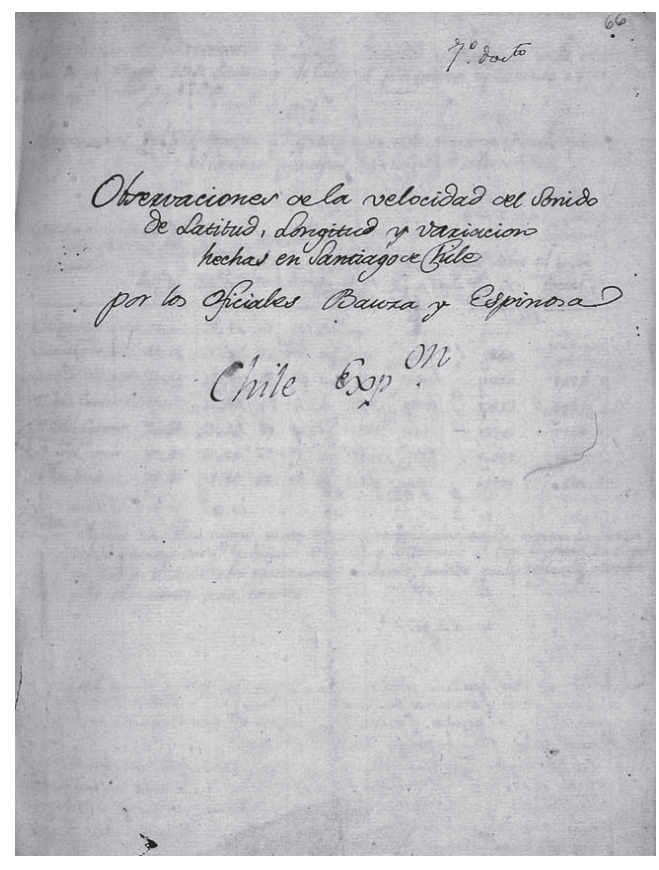

Fuente: Archivo Museo Naval de Madrid

Como se advierte en las primeras páginas de esta obra, la incorporación de estas experiencias como anexo obedeció al propósito de entregar a los lectores "muchas curiosas y apreciables noticias tocantes á la náutica, á la geografía, ó al mérito y trabajos poco conocidos de algunos marinos españoles" (Memorias, 1809, Tomo I: 1). A su vez, José Espinosa en la introducción de la Memoria Segunda, señalaba que si bien uno de los objetos principales de la empresa comandada por Alejandro Malaspina había sido el "cumplimiento cabal" de las tareas hidrográficas, también eran importantes "otras observaciones" en que interesaban mucho "la geografía física y la náutica, como son las del péndulo invariable, las de la variación é inclinación de la aguja, las de las mareas, las de la velocidad del sonido, y las del barómetro y termómetro aplicadas á la averiguación de las diferencias del nivel de los lugares en que se executaron", puesto que "teniéndolas todas reunidas" los lectores podrían comprender "las conseqüencias ó resultados generales á que den lugar, y hacerse las comparaciones que convegan" (Memorias, 1809: XXIX y XXX). De esa forma, Espinosa como editor consideró necesaria su publicación por la virtud que las mismas tenían para el adelanto de la Física, puesto que al ser publicadas y con eso entregarles la posibilidad de ser cotejadas con otras experiencias de similares características, hacía que los resultados obtenidos en la colonia más austral del imperio español entraran dentro de la discusión sobre el problema de la velocidad del sonido.

Prueba de lo anterior fue la valoración que de estas hizo Alejandro Malaspina. Este cuando recibió los trabajos sobre las experiencias realizadas señaló: "Con la primera ocasión oportuna remitiré al Exmo Sr Ministro de Marina, las observaciones echas en Santiago, que $V m s$ se han servido en embiarne seguramente para mi admiración, mas bien que aprobación; pues no necesita de esta última, quanto acredite un Talento, y un Celo poco comunes, y si deve tributarse la otra a unas Operaciones ejecutadas con tanta exactitud y laboriosidad, como lo indica el mismo resumen. No dexará pues S.M (como lo espero) de mirar este trabajo, como una prueba de la constante aplicación de $V \mathrm{~ms}$ en qualesquiera tiempos, y circunstancias" (AMN, Ms. 427, Doc. 56).

Las palabras de Malaspina reflejan la utilidad de dar a conocer estos avances con la mayor rapidez posible a las altas autoridades españolas, ya que bajo su parecer aportaban nuevas ideas en el ámbito científico que eran dignas de reconocimiento por parte de la monarquía, lo que demuestra que el modelo de relaciones entre los científicos y el Estado estaba condicionado por la manera en que este último promovía la ciencia y en su decisión de señalar qué conocimientos eran ventajosos para la nación y cuáles no. Además, deja entrever la importancia cada vez mayor que tiene el Estado en los procesos de construcción y validación del conocimiento científico (Hernández, 2008: 31 y 135). Pero no solo eso, Malaspina además apreciaba la práctica y experiencia de ambos oficiales, destacando sus cualidades profesionales y humanas en un trabajo que justificaba como actividad de la exploración científica en América. 
En 1817, dos años después de la muerte de Espinosa, el texto fue nuevamente publicado, esta vez en francés, bajo el título "Observations sur la vitesse du son faites á Santiago du Chili" en los Annales de Chimie et de physique. Considerada una de las revistas científicas francesas de mayor prestigio en la difusión de los progresos químicos y físicos, la aparición de los estudios hechos por Espinosa y Bauzá en este órgano especializado, confirmaban el interés despertado en la comunidad científica internacional por estas investigaciones, debido a su singularidad de ser las únicas experiencias de este tipo practicadas en una zona tan periférica y apartada como lo era en ese entonces la ciudad de Santiago.

Aunque el texto aparecido no fue más que una traducción textual del original en español, y que debe haber sido Felipe Bauzá quien hiciera los trámites para su publicación, aquella transferencia nos habla del reconocimiento que el mundo científico internacional hizo a las mediciones de la velocidad del sonido en Santiago en 1794. Pero más importante todavía, demuestra que estos trabajos seguían teniendo vigencia en el ámbito de las ciencias luego de más de veinte años de realizadas las observaciones, y casi diez desde la primera vez que estos resultados fueran comunicados al público.

El alcance de este trabajo fue aún mayor, puesto que en 1819 volvió a recogerse en $\mathrm{Co}^{-}$ rrespondence, astronómique, geographique, hidrographique et statistique du baron de Zach (Cuesta, 1992: 831). Además, en 1828 el astrónomo alemán Jabbo Oltmanns publicó en el Journal für die reine und angewandte Mathematik -una de las revistas más importantes de matemáticas en alemán- un artículo en el cual exponía el experimento hecho por Espinosa y Bauzá en 1794, contrastando y analizando los resultados obtenidos por ambos oficiales españoles con las observaciones sobre la velocidad del sonido practicadas por diversos científicos anteriores y posteriores a las hechas en "Ios inconmesurables Ilanos de América del Sur" (Oltmanns, 1828: 308, 309-311).

Como lo destaca Oltmanns, este conocía otras observaciones y fragmentos del viaje "memorable" que ambos marinos habían realizado desde Valparaíso a Buenos Aires, sin embargo, le parecía necesario divulgar estas experiencias sobre la velocidad del sonido, "de dos de los más hábiles astrónomos españoles", puesto que eran las primeras realizadas en América del Sur, advirtiendo que el método de observación utilizado fue muy similar al aplicado en los alrededores de París por Arago, Gay-Lussac, Bouvard, Prony, Mathieu y Humboldt en 1822, mediciones que no solo tendían a extremar las posibilidades de obtener un valor más exacto, sino que también buscaban verificar experimentalmente la formula teórica de la velocidad entregada por el matemático francés Pierre Simon de Laplace (Oltmanns, 1828: 308; Pla, 21).

Desde ese punto de vista, la investigación de Espinosa y Bauzá para el astrónomo alemán no constituía "meramente una curiosidad científica", sino que subrayaba su alcance y ventaja en materias de navegación y observaciones geodésicas, ya que se podía usar el valor de la velocidad del sonido para concluir ciertas distancias, y así aplicarse esta práctica a la determinación de las bases necesarias para el levantamiento de planos, entre otras cuestiones (Oltmanns, 1828: 312; Juan y Ulloa, 1768: 142).

El hecho de que estas observaciones realizadas en Chile hayan sido difundidas en círculos científicos europeos, a su vez, pone el acento en el análisis de la actividad científica llevada a cabo en regiones periféricas. Siguiendo a Marcos Cueto, el experimento sobre la velocidad del sonido al ser narrado y dado a conocer a la comunidad científica permitió a los oficiales españoles no solo generar un saber en una zona "marginal del acervo mundial del conocimiento" (Cueto, 1989: 28), sino también entrar a una red científica que les dio la posibilidad de dar credibilidad a las experiencias hechas. En ese sentido, la interacción de un trabajo moderno sobre propagación de las ondas sonoras -familiarizado con un lenguaje propio de la ciencia ilustrada y en un marco de referencia sobre autores que con anterioridad estudiaron este fenómeno (Espinosa y Bauza, 1809: 170; AMN, Ms. 579, f.150, 150v., 153)-, en un contexto cultural "supuestamente tradicional y "periférico" a los centros mundiales de la ciencia" (Cueto, 1989:29), no hace más que evidenciar la coincidencia 
de tradiciones científicas e instrumentales desiguales a la hora de comprender el quehacer científico en esta región. Por lo tanto, y como lo veremos a continuación, la incorporación de saberes locales dentro de sistemas y marcos de referencias ilustrados, implicó que el conocimiento elaborado por Espinosa y Bauzá fuese consecuencia igualmente del vínculo que estos sostuvieron con los habitantes del lugar, quienes prestaron la ayuda necesaria para llevar con éxito las mediciones y observaciones sobre la velocidad del sonido en América meridional.

\section{Experiencias del sonido en la periferia}

La renovación que desde hace algún tiempo han experimentado los estudios sobre historia de la ciencia, gracias a los aportes de la historia social y la historia cultural, y al diálogo con la sociología del conocimiento, significó que se cuestionaran los principios teóricos de la disciplina que en sus orígenes solo intentaba rescatar y reconstruir las grandes epopeyas científicas de significación mundial, centrándose en la vida de científicos eminentes y sus descubrimientos, con el propósito de legitimar y enaltecer el método y los logros de la ciencia occidental ( $\mathrm{Pi}$ mentel, 2010; Galison, 2008; Moro, 2005; Nieto, 1995; Barona, 1994). De esa forma, la disciplina abrió su campo de investigación hacia el estudio de la actividad científica en regiones culturales antes excluidas, emergiendo reflexiones en torno a los procesos de fabricación y legitimación del conocimiento en la periferia, es decir, en los países alejados de los centros científicos internacionales (Cueto, 1989: 21; Saldaña, 1996: 14-15).

En el ámbito colonial americano, la producción del conocimiento científico se desarrolló en el marco de tradiciones culturales en las cuales ya existían complejas relaciones entre la naturaleza y la sociedad, quedando en evidencia que el trabajo del científico europeo no fue solo resultado de su relación directa con el mundo natural, sino que constituyó un esfuerzo donde la interacción con el saber local fue clave a la hora de generar nuevos conocimientos (Nieto, 2009 y 2010; Cueto, 1989:28-30; Sagredo y González, 2004).
La participación activa de criollos e indígenas en la reflexión sobre la naturaleza americana y sus fenómenos, significó tanto que estos no estuvieran aislados de los movimientos científicos europeos, como que el quehacer científico europeo se viera beneficiado por el contacto entre ambos (Soto, Puig-Samper, y González-Ripoll (eds.), 1999 y 2003). Ejemplo de lo indicado fue que Espinosa y Bauzá una vez instalados en la ciudad de Santiago, solicitaron "de algunos sujetos curiosos los Instrumentos astronómicos y físicos" para llevar a cabo con éxito sus observaciones. Así señalan que Manuel Pérez Cotapos, destacado político y comerciante, les facilitó "un anteojo de Dolland de 9 pies de largo y sin pie". Además recibieron del arquitecto Joaquín Toesca "un buen teodolite inglés del Artista Colle que nos ha servido de quarto de círculo y con este instrumento tomamos casi diariamente alturas del sol y azimutes magnéticos" (AMN, Ms. 579, f. 154). Aquella intersección entre ciencia local y ciencia europea en escenarios periféricos, por lo tanto, demuestra cómo la colaboración colonial fue fundamental en la producción científica imperial, pues según el ejemplo entregado, el instrumental con que contaron los oficiales españoles para hacer sus investigaciones en una región como Chile, obedeció a la relación que estos sostuvieron con algunos vecinos del lugar, siendo ellos los que les permitieron el acceso a artefactos que no poseían.

Por otra parte, es posible apreciar que Espinosa y Bauzá al comenzar a relatar el experimento sobre la velocidad del sonido en Chile, mencionan a pie de página la relevancia que tuvo en estas observaciones el auxilio del criollo de origen peruano Miguel de Lastarria: "Don Miguel de Lastarria, vecino de Santiago, sugeto muy aplicado y amante de las ciencias, nos acompañó á estas distintas operaciones; y con su conocimiento del país y sus proporciones en él nos fue muy útil" (Espinosa y Bauzá, 1809: 173). Igualmente, en una de las copias de las "Observaciones..." se lee luego de cada una de las experiencias realizadas y anotadas prolijamente por los oficiales la frase: "Don Miguel de Lastarria contó tiempo iguales" (AMN, Ms. 579, f. 87-88). No resulta extraño que fuera Lastarria quien ayudara a los marinos a concluir sus investigaciones, pues como discípulo en ciencias exactas y naturales del cosmógrafo 
Gabriel Moreno en la Universidad de San Marcos, fue nombrado una vez llegado a Santiago catedrático de estas materias en el Convictorio Carolino. Como lo destacaba Benjamín Vicuña Mackenna: "El doctor Lastarria, dictando filosofía, enseñaba al mismo tiempo aritmética, geometría, estática, hidrostática, maquinaria, geografía, cosmografía, historia y cronología" donde "gentes por particular gusto y complacencia corrían á oir sus lecciones". Sin embargo, al poco andar las innovaciones en conocimientos científicos introducidas por Lastarria fueron vistas con recelo por los delegados de la Inquisición en Santiago, siendo clausurada a mediados de 1786 su cátedra y separado de sus funciones (Noticias biográficas de Don Miguel J. de Lastarria, 1879: 5-9). Una vez asumido como gobernador Tomás Álvarez Acevedo, y aprovechando los conocimientos que Lastarria poseía en Física, le solicitó que aplicara aquellos saberes a dar impulso a la industria minera chilena (Noticias biográficas de Don Miguel J. de Lastarria, 1879: 11).

De esa forma, Espinosa y Bauzá al momento de hacer las observaciones sobre la velocidad del sonido, no solo contaron con la experiencia y erudición de Lastarria, sino que según su relato este tuvo una intervención activa en las mismas. Asimismo, la participación del criollo fue esencial a la hora de corroborar los datos obtenidos por los científicos españoles en la medición de tiempo transcurrido entre que era vista la explosión del cañón y se oía el disparo, desde un paraje a otro, pues este también hizo sus propias medidas, convirtiéndose aquella información entregada por Lastarria en garante de los propios resultados de Espinosa y Bauzá. En ese sentido, la actitud pasiva que normalmente se le atribuye al sujeto americano en el proceso de producción de conocimiento en las posesiones ultramarinas, queda descartada, ya que el quehacer científico como queda demostrado, es efecto tanto del trabajo de los científicos como de los antecedentes expuestos por los anfitriones (Sagredo y González, 2004: 28).

Ahora bien, respecto a las reacciones locales que pudo tener el experimento llevado a cabo por Espinosa y Bauzá en Santiago a finales del siglo XVIII, no hemos encontrado noticia alguna que nos señale sus efectos $y$ repercusiones en el desarrollo científico local. Si bien antes de la creación del Instituto Nacional en 1813, en Chile solo hubo actividades esporádicas en el campo de la Física, creemos que el estudio de las experiencias sobre la velocidad del sonido practicadas en Santiago en 1794, contribuye a la comprensión de la actividad científica en la región y abre paso a nuevas interrogantes al respecto. Asimismo, pensamos que cuestiona la idea de la inexistencia de producción científica en Chile en la centuria dieciochesca, quedando demostrado que el estudio presentado entrega la posibilidad de investigar un campo poco explorado por la historiografía nacional (Gutiérrez y Gutiérrez, 2006: 480).

Por lo tanto, a través del estudio de las experiencias sobre la velocidad de sonido en Chile, hemos querido dar a conocer un caso concreto sobre la forma en que se aplicó y generó el conocimiento durante el proceso de reconocimiento y dominación de los territorios ultramarinos por el Estado español. En este contexto, el estudio de las prácticas científicas no solo enriquece la mirada sobre la relación entre imperio y conocimiento, sino que ayuda a la comprensión de la producción científica en la periferia, ampliando nuestra percepción sobre problemas tales como la elaboración, desarrollo y práctica de la ciencia colonial en América.

\section{Referencias bibliográficas}

ARCHIVO MUSEO NAVAL, Ms. 314, f. 183; Ms. 427, Doc. 56; Ms. 579, f. 59, 59v., 63, 69, 71, 71 v., 72, 87, 88, 150, 150v., $152 \mathrm{v} ., 153$ y 154 .

AA.VV. De la Ciencia llustrada a la Ciencia Romántica. Actas de las II jornadas sobre "España y las expediciones científicas en América y Filipinas". Aranjuez: Doce CallesAteneo de Madrid, 1995.

AA.VV. La ciencia española en ultramar. Actas de las I Jornadas sobre "España y las expediciones científicas en América y Filipinas". Aranjuez: Doce Calles-Ateneo de Madrid, 1991.

ALCALÁ, D. Memorias sobres las observaciones de la latitud y longitud en el Mar. Madrid: Imprenta de Ibarra, 1796. 
AMUNÁTEGUI, M. L. Los precursors de la Independencia de Chile. Tomo Tercero. Santiago de Chile: Imprenta de la "República" de Jacinto Núñez, 1872.

BARONA, J. Ciencia e historia: Debates y tendencias en la historiografía de la ciencia. Valencia: Seminari d'Estudis sobre la Ciència, 1994

BEYER, R. T. Sounds of our times. Two hundred years of acoustics. New York: AIP press/Springer, 1999.

CUESTA, P. José Espinosa y Tello y su aportación a la historia de la hidrografía. Tomo 2. Madrid: Departamento de Historia Moderna y Contemporánea, Facultad de Geografía e Historia, Universidad Complutense de Madrid, 1992.

CUETO, M. Excelencia científica en la periferia. Actividades científicas e investigación biomédica en el Perú. 1890-1950. Lima: GRADE, 1989.

DE MENDOZA, J. Tratado de navegación por Don Josef de Mendoza y Ríos, Teniente de Navío de la Real Armada, Tomo Segundo. Madrid: Imprenta Real, 1787.

ESPINOSA, J. y BAUZÁ, F. Observaciones de la velocidad del sonido, de latitud, longitud y variación hechas en Santiago de Chile por el Teniente de Navío Don Josef de Espinosa y el Alférez de Navío Don Felipe Bauzá en 1794. En: Memorias sobre las observaciones astronómicas hechas por los navegantes españoles en distintos lugares del globo; las quales han servido de fundamento para la formación de las cartas de marear publicadas por la Dirección de Trabajos Hidrográficos de Madrid: Ordenadas por Don Josef Espinosa y Tello, Tomo I. Segunda Memoria. Madrid: Imprenta Real, 1809, p. 169-182.

ESPINOSA, J. y BAUZÁ, F. Observations sur la vitesse du son faites à Santiago du Chili. Annales de Chimie, N 7, 1817, p. 93-99.

ESTRELLA, E.; PIMENTEL, J. y HIGUERAS, D. La Expedición Malaspina 1789-1794. Trabajos zoológicos, geológicos, químicos y físicos en Guayaquil de Antonio Pineda Ramírez.
Madrid: Ministerio de Defensa, Museo Naval y Lunwerg Editores, 1996, Tomo VIII.

GALERA, A. La ilustración española y el conocimiento del Nuevo Mundo. Las Ciencias Naturales en la Expedición Malaspina (17891794): la labor científica de Antonio Pineda. Madrid: CSIC, 1988.

GALERA, A. Ciencia a la sombra del Vesubio. Ensayo sobre el conocimiento de la naturaleza. Madrid: CSIC, 2003.

GALINSON, P. Ten problems in History and Philosophy of Science. Isis, 2008, N 99, p. 111-124.

GARRIDO, A. Física del Sonido. Madrid: Sanz y Torres, 1996.

GAY, P. La edad de las luces. México: Ediciones Culturales Internacionales, 1983.

GLICK, T., Imperio y dependencia científica en el siglo XVIII español e inglés: la provisión de los instrumentos científicos. En: PESET, J. Ciencia, vida y espacio en Iberoamérica. Madrid: CSIC, 1989.

GONZÁLEZ, J. Primeros levantamientos cartográficos generales de Chile con base científica: Los mapas de Claudio Gay y Amadeo Pissis. Revista de Geografía Norte Grande, 2007, No38, p. 21-44.

GRANVILLE, W. Trigonometría plana y esférica con tablas trigonométricas. México: Unión Tipográfica Editorial Hispano-Americana, 1950.

GUTIÉRREZ, C. y GUTIÉRREZ, F. Física: su trayectoria en Chile (1800-1960). Historia, 2006, N³9, p. 477-496.

HERNÁNDEZ, R. El matemático impaciente. La Condamine, las pirámides de Quito y la ciencia ilustrada (1740-1751). Lima: IFEA, Universidad Andina Simón Bolívar, IEP, 2008.

HIGUERAS, M.D. Catálogo crítico de los documentos de la Expedición Malaspina (1789-1794) del Museo Naval. Madrid: Museo Naval, 1987. 
JUAN, J. y ULLOA, A. Observaciones astronómicas y phisicas hechas de orden de $S$. Mag. en los Reynos del Peru; de las quales se deduce la figura, y magnitud de la Tierra y se aplica a la navegación. Madrid: Juan de Zuñiga, 1768.

LA CONDAMINE. Journal du voyage fait par ordre du roi à l' equateur, servant $d^{\prime}$ introduction historique à la mesure des trois premieres degrés du meridien. París: L'Imprimerie Royale, 1751.

LAFUENTE, A. y DELGADO, A.J. La geometrización de la Tierra: Observaciones y resultados de la expedición geodésica hispanofrancesa al Virreinato del Perú (1735-1744). Madrid: CSIC, 1984.

LAFUENTE, A. y VALVERDE, N. Los mundos de la ciencia en la Ilustración española. Madrid: Fundación Española para la Ciencia y la Tecnología, 2003

Memorias sobre las observaciones astronómicas hechas por los navegantes españoles en distintos lugares del globo; las cuales han servido de fundamento para la formación de las cartas de marear publicadas por la Dirección de Trabajos Hidrográficos de Madrid: Ordenadas por Don Josef Espinosa y Tello, Tomo I. Madrid: Imprenta Real, 1809.

MORO, O. La nueva historia de la ciencia y la sociología del conocimiento científico: Un ensayo historiográfico. Asclepio, Vol. LVII, 2005, p. 255-280.

NEWTON, I. Principios Matemáticos de la Filosofía Natural. Madrid: Tecnos, 1987.

NIETO, M. Poder y conocimiento científico. Nuevas tendencias en historiografía de la ciencia. Historia Crítica, $N^{\circ} 10,1995$, p. 3-13.

NIETO, M. Remedios para el Imperio. La historia natural y la apropiación del nuevo mundo. Colombia: Editorial de la Universidad de los Andes, 2006.

NIETO, M. Orden natural y orden social: Ciencia y política en el Semanario del Nuevo Reyno de Granada. Bogotá: Uniandes-Ceso, 2009.
NIETO, M. Serpientes, venenos y remedios: saberes locales y la ciencia de los ilustrados de la Nueva Granada. En: SAGREDO, R. (editor) Ciencia-Mundo. Orden republicano, arte y nación en América. Santiago de Chile: Centro de Investigaciones Diego Barros Arana, Editorial Universitaria, 2010, p. 97119.

Noticias biográficas de Don Miguel J. de Lastarria. Montevideo: Imprenta a Vapor de la Nación, 1879.

NOVO Y COLSON, P. Viaje político-científico alrededor del Mundo por las corbetas Descubierta y Atrevida al mando de los capitanes de navío don Alejandro Malaspina y don José de Bustamente y Guerra desde 1789 a 1794. Madrid: Imprenta de la Viuda e hijos, 1885.

OLTMANNS, J. Beobachtungen über die Geschwindigkeit des schalls in den Ebenen Süd-Americas, angestellt von Espinosa und Bauza. Journal für die reine und angewandte Mathematik, Vol. 2, 1828, p. 307-316.

PESET, J.L. y LAFUENTE, A. Carlos III y la ciencia de la Ilustración. Madrid: Alianza Editorial, 1989.

PIMENTEL, J. La física de la Monarquía. Ciencia y Política en el pensamiento colonial del Alejandro Malaspina (1754-1810). Madrid: Doce Calles, 1998.

PIMENTEL, J. ¿Qué es la historia cultural de la ciencia? Arbor, 2010, N 743, p. 417424.

REAL ACADEMIA ESPAÑOLA. Diccionario de la Lengua Española, 21 a ed. Tomo I. Madrid: RAE, 1992.

REVISTA DE INDIAS. Número monográfico sobre las expediciones ilustradas, 1987, $\mathrm{N}^{\circ} 180$.

SAGREDO, R. y GONZÁLEZ, J.I. La EXpedición Malaspina en la frontera austral del imperio español. Santiago de Chile: Centro de Investigaciones Diego Barros Arana y Editorial Universitaria, 2004. 
SAGREDO, R. Navegación científica en el Mar del Sur. El piloto Moraleda (1772-1810). Revista de Historia Iberoamericana, 2008. Disponible en internet: http:// revistahistoria.universia.cl/pdfs_revista/articulo_89_1250114582587.pdf.

SALAZAR, L. Discurso sobre los progresos y estado actual de la hidrografía en España. Memorias sobre las observaciones astronómicas hechas por los navegantes españoles en distintos lugares del globo; las quales han servido de fundamento para la formación de las cartas de marear publicadas por la Dirección de Trabajos Hidrográficos de Madrid: Ordenadas por Don Josef Espinosa y Tello, 1809, Tomo I, p. 1-113.

SALDAÑA, J.J. (coordinador). Historia social de las ciencias en América Latina. México: Porrúa, 1996.

SELLÉS, M. Historia de la ciencia y de la técnica. Astronomía y navegación en el siglo XVIII. Madrid: Akal, 1992.
SELLÉS, M. Navegación astronómica en la España del siglo XVIII. Madrid: Universidad Nacional de Educación a Distancia, 2000.

SHAPIN, S. y SCHAFFER, S. El Leviathan y la bomba de vacío. Hobbes, Boyle y la vida experimental (1985). Buenos Aires: Universidad Nacional de Quilmes, 2005.

SHURMANN, P. Historia de la Física, Tomo II. Buenos Aires: Editorial Nova, 1946.

SOTO, D.; PUIG-SAMPER, M. y GONZÁLEZ-RIPOLL, M. (editores). Científicos criollos e Ilustración. Madrid: Doce Calles-Colciencias-Rudecolombia, 1999.

SOTO, D.; PUIG-SAMPER, M. y GONZÁLEZ-RIPOLL, M. (editores). Recepción y difusión de textos ilustrados. Madrid: Doce Calles, 2003.

VALVERDE, N. Actos de precisión. Instrumentos científicos, opinión pública y economía moral en la llustración Española. Madrid: CSIC, 2007. 